\title{
Abbreviations
}

- Journal titles are abbreviated according to the Année Philologique.

- Abbreviations of ancient authors and works are those of the Oxford Classical Dictionary $(O C D)$ 4th ed., supplemented, when needed, by Liddell Scott Jones Greek-English Lexicon $(L S /)$, and the Oxford Latin Dictionary (OLD).

- Abbreviations of medical authors (Corpus Hippocraticum and Corpus Galenicum) are those of the Corpus Medicorum Graecorum/Latinorum (Berlin-Brandenburgische Akademie der Wissenschaften).

- Seneca's Dialogi are not quoted by number but by title, abbreviated thus:

\section{Seneca's Dialogi}

Brev.

De brevitate vitae (Dialogorum lib. $X$ )

Helv.

Ad Helviam matrem de consolatione (Dialogorum lib. XII)

Ir.

De ira (Dialogorum libri III-V)

Marc.

Ad Marciam de consolatione (Dialogorum lib. VI)

Ot.

De otio (Dialogorum lib. VIII)

Polyb.

Ad Polybium de consolatione (Dialogorum lib. XI)

Prov.

De providentia (Dialogorum lib. I)

Tranq. an. De tranquillitate animi (Dialogorum lib. IX)

VB De vita beata (Dialogorum lib. VII)

\section{Abbreviations from the Corpus Hippocraticum}

\begin{tabular}{|c|c|}
\hline De Arte & 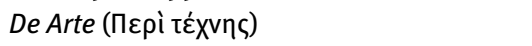 \\
\hline Epid. & 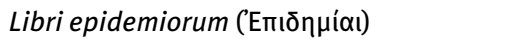 \\
\hline Medic. & 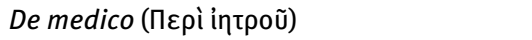 \\
\hline Vict. & 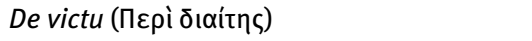 \\
\hline$V M$ & 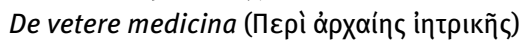 \\
\hline
\end{tabular}

\section{Abbreviations from the Corpus Galenicum}

\begin{tabular}{|c|c|}
\hline$C C$ & 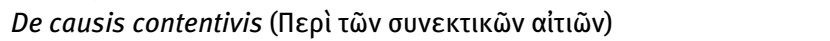 \\
\hline Diff. Resp. & 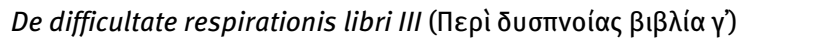 \\
\hline Dign. Puls. & 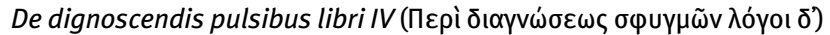 \\
\hline Loc. Aff. & 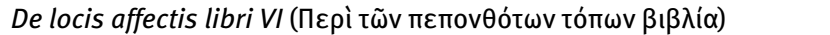 \\
\hline Meth. Med. & 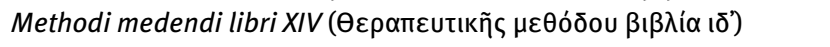 \\
\hline Mot. Musc. & 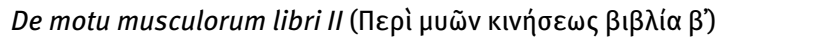 \\
\hline PHP & 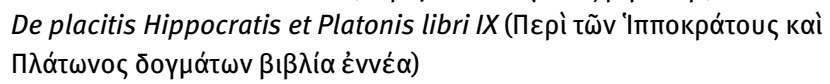 \\
\hline
\end{tabular}

\section{Journals}

AAntHung

Acta antiqua Academiae Scientiarum Hungaricae

AntPhilos

Antiquorum Philosophia

$A B G$

Archiv für Begriffs geschichte

$A C$

L'Antiquité classique

A\&A

Antike und Abendland

$A J P h$

The American Journal of Philology

https://doi.org/10.1515/9783110673715-204 


\begin{tabular}{|c|c|}
\hline$B A G B$ & Bulletin de l'Association Guillaume Budé \\
\hline BJHP & British Journal for the History of Philosophy \\
\hline BStudLat & Bollettino di Studi Latini \\
\hline Bull Hist Med & Bulletin of the History of Medicine \\
\hline Cogn Psychol & Cognitive Psychology \\
\hline$C P h$ & Classical Philology \\
\hline$C Q$ & The Classical Quarterly \\
\hline$C R$ & The Classical Review \\
\hline$E L$ & Études de lettres \\
\hline$G \& R$ & Greece and Rome \\
\hline GIF & Giornale italiano di filologia \\
\hline$H S P h$ & Harvard Studies in Classical Philology \\
\hline J Aesthet and & The Journal of Aesthetics and Art Criticism \\
\hline Art Crit & \\
\hline ICS & Illinois Classical Studies \\
\hline JHS & The Journal of Hellenic Studies \\
\hline JTLA & Journal of the Faculty of Letters of the University of Tokyo \\
\hline$L E C$ & Les Études Classiques \\
\hline$M A A R$ & Memoirs of the American Academy in Rome \\
\hline MAT & Memorie della Accademia delle Scienze di Torino \\
\hline$M D$ & Materiali e discussioni per l'analisi dei testi classici \\
\hline MH & Museum Helveticum \\
\hline NNG & Machrichten von der Gesellschaft der Wissenschaften zu Göttingen \\
\hline PhilosAnt & Philosophie Antique \\
\hline Proc Aristot Soc & Proceedings of the Aristotelian Society \\
\hline$R C C M$ & Rivista di cultura classica e medioevale \\
\hline REL & Revue des études latines \\
\hline RFIC & Rivista di filologia e di istruzione classica \\
\hline$R h M$ & Rheinisches Museum für Philologie \\
\hline RHR & Revue de l'Histoire des Religions \\
\hline SIFC & Studi Italiani di Filologia classica \\
\hline TAPhA & Transactions of the American Philological Association \\
\hline Trends Cogn Sci & Trends in Cognitive Science \\
\hline
\end{tabular}

\section{Further abbreviations in alphabetical order}

ANRW Temporini, H. (ed.) Aufstieg und Niedergang der römischen Welt. Berlin, 1972-.

DK Diels, H./Kranz, W. (eds.) Die Fragmente der Vorsokratiker. Zürich, $1952^{6}$. (Originally published 1903).

EK Edelstein, I./Kidd, G. (eds.) Posidonius, i-iii. Cambridge, 1972-99.

K Kühn, K.G. (ed.) Medicorum Graecorum Opera Quae Exstant, 1-20. Leipzig, 1821-1833.

M Marx, F. (ed.) C. Lucilii Carminum Reliquiae. Leipzig, 1904.

PIR $\quad$ Prosopographia Imperii Romani saec. I. II. III. 2nd ed. Berlin, 1933-.

RAC Klauser, T. (ed.) Das Reallexikon für Antike und Christentum und das F. J. Dölger-Institut in Bonn. Berichte, Erwägungen, Richtlinien, i. Stuttgart, 1950. 
$R E$

SVF

ThlL

Trypho fig.
Paulys Real-Enzyklopädie der klassischen Altertumswissenschaft.

Stuttgart, 1894-1980.

von Arnim, H. (ed.) Stoicorum veterum fragmenta. i-iii. Leipzig, 1903-

1905. (quoted by volume and fragment number)

Thesaurus linguae Latinae. Leipzig, 1900-.

Trypho, De figuris, in Spengel, L. (ed.) Rhetores Graeci, iii. Leipzig,

1856. 
